\title{
Precision Imaging of Frequency Stepped SAR with Frequency Domain Extracted HRRP and Fast Factorized Back Projection Algorithm
}

\author{
Can-bin Yin and Da Ran \\ Equipment Academy, Beijing 101416, China \\ Correspondence should be addressed to Can-bin Yin; expressesp@126.com
}

Received 3 September 2016; Accepted 20 December 2016; Published 16 February 2017

Academic Editor: Herve Aubert

Copyright (C) 2017 Can-bin Yin and Da Ran. This is an open access article distributed under the Creative Commons Attribution License, which permits unrestricted use, distribution, and reproduction in any medium, provided the original work is properly cited.

\begin{abstract}
Novel frequency domain extracted method (FDEM) to obtain high range resolution profile (HRRP) for frequency stepped synthetic aperture radar (SAR) is proposed in this paper, and the mathematical principle and formulas of this new HRRP obtaining idea combined with classical fast Fourier transform (FFT), chirp $z$ transform (CZT), and single point Fourier transform (SPFT) are deduced, analyzed, and compared in detail. Based on the HRRP data, precision imaging processing is completed using a data block partition based fast factorized back projection algorithm. Imaging validations are executed and all results proved that the FDEM has a great capability of antijamming. It is more effective than conventional time domain IFFT method (TDM) and shows a great promise in frequency stepped radar imaging and applications.
\end{abstract}

\section{Introduction}

Apart from the classical linear frequency modulated (LFM) waveform widely used in modern radar systems, the frequency stepped waveform also acts as an important kind of frequency-coded radar signal for high range resolution profile (HRRP) obtaining [1-22]. Besides providing the ability of synthesizing high resolution range profiles, which improves the range accuracy, reduces the amount of clutter within resolution cell, reduces multipath, and provides HRRP and aids in target classification, it can also make targets become visible above the residual clutter and noise along with clutter cancellation, which plays an important role in the detection of low radar cross section (RCS) targets embedded in ambience noise. Radars employing a frequency stepped waveform increase or decrease the frequency successively through pulses in one burst. It can be viewed as an interpulse modulated pulse compression waveform in which modulation is applied across the pulses instead of within individual pulses and provides a high range resolution capability by producing a detailed target range profile and a detailed two-dimensional image of the target when coupled with
SAR/ISAR. Frequency stepped radar's HRRP and 2D images are used for target recognition and classification. Currently, the fine range resolution capability of frequency stepped radar is being exploited to solve the difficult problem of detection of high-speed, low-RCS targets in the presence of large clutter. This class of problems includes detection of cruise missiles, sea skimming antiship missiles, and stealth aircraft. Compared to classical LFM based radar, frequency stepped radar has a narrow instantaneous bandwidth but attains a large effective bandwidth sequentially over many pulses in the processor. As a result, the hardware requirements become less stringent that lower-speed A/Ds (commensurate with the low bandwidth of individual pulses) and slower processors can be used. Besides, the receiver bandwidth is much smaller, resulting in lower noise bandwidth and a higher signalto-noise ratio (SNR). Another important but less obvious advantage of frequency stepped radar is the rejection of multiple components around clutter, which can be quite large for high-PRF waveforms. Due to different frequencies of successive pulses, clutter from ambiguous ranges will come at frequencies other than the one from the target area and most of the ambiguous clutter returns which lie outside the pass 


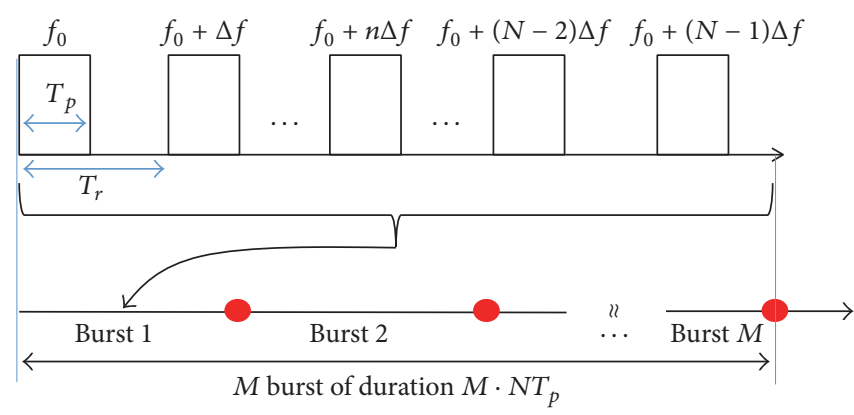

FIgURE 1: A frequency stepped waveform.

band will be rejected by the receiver IF amplifier. Apart from enhancement of SNR, it will reduce the dynamic range of the received return, which in turn reduces the number of bits per sample requirement of $\mathrm{A} / \mathrm{D}$.

In this paper, the basic principles of frequency stepped radar and conventional time domain IFFT method (TDM) for range profile obtaining are firstly reviewed in Section 2; in Section 3, the drawbacks of conventional TDM are analyzed and a novel frequency domain extracted method (FDEM) to obtain HRRP for frequency stepped synthetic aperture radar (SAR) is proposed. Based on the idea in [23], the mathematical principle and formulas of FDEM based HRRP obtaining idea combined with classical fast Fourier transform (FFT), chirp $z$ transform (CZT), and single point Fourier transform (SPFT) are detailed deduced, analyzed, and compared using both virtual and real radar data. Based on the HRRP data, a precision data block partition based fast factorized back projection algorithm for synthetic aperture imaging is given. Finally, the imaging validations are executed in Section 4 and conclusions are made in Section 5.

\section{Frequency Stepped Radar Principles}

The frequency stepped waveform consists of a group of $N$ coherent pulses frequencies increase from pulse to pulse by a fixed frequency increment as shown in Figure 1. The frequency of the $i$ th pulse can be written as

$$
f_{i}=f_{0}+b_{i} \Delta f
$$

where $f_{0}$ is the starting carrier frequency, $\Delta f$ is the frequency increment, $T_{p}$ is the pulse width, and the time interval $T_{r}$ between pulses is adjusted for ambiguous or unambiguous range. The frequency stays constant within each pulse. Groups of $N$ pulses, also called a burst, are transmitted and received before any processing is initiated to realize the highresolution potential of this waveform. The burst time, that is, the time corresponding to transmission of $N$ pulses, is called coherent processing interval (CPI) as in conventional radars. $\left\{b_{i}=i, i=1,2,3, \ldots, N\right\}$ is the frequency code used in radar system, and the waveform is known as frequencycoded signal if $\left\{b_{i}\right\}$ is a random sequence. For individual pulse, its bandwidth is approximately equal to the inverse of pulse width. Pulses of typical time duration have narrow bandwidth, which makes the instantaneous bandwidth of the radar narrow. Thus, narrowband equipment (except for the antenna and transmitter) can be used. However, as explained later, effective large bandwidth can be realized by appropriately processing $N$ pulses in a CPI, and the effective bandwidth is determined by the total frequency excursion, that is, $N \Delta f$ over the duration of $N$ pulses. The range resolution of frequency stepped radar is determined by

$$
\rho_{r}=c / 2 N \Delta f
$$

Suppose the $i$ th transmitted pulse of radar is

$$
s_{t}(t)=A_{i} \exp \left(j 2 \pi f_{i} t+\theta_{i}\right), \quad i T_{r} \leq t \leq i T_{r}+T_{p},
$$

where $A_{i}$ is the pulse amplitude, $f_{i}$ is the carrier frequency, $\theta_{i}$ is the initial phase, and the echo can be expressed as

$$
\begin{aligned}
s_{r}(t)=A_{i}^{\prime} \exp \left[j 2 \pi f_{i}\left(t-\tau_{i}\right)+\theta_{i}\right] & \\
& i T_{r}+T_{p} \leq t \leq i T_{r}+T_{p}+\tau_{i},
\end{aligned}
$$

where $A_{i}^{\prime}$ is the amplitude and $\tau_{i}=2 R / c$ is the round trip delay of the $i$ th pulse determined by the distant $R$ between radar and target. Suppose the reference signal is

$$
s_{\text {ref }}(t)=A \cdot \exp \left(j 2 \pi f_{i} t+\theta_{i}\right), \quad i T_{r} \leq t \leq(i+1) T_{r} .
$$

Set the sampling time as $i T_{r}+T_{p} / 2+2 R / c$, and the intermediate frequency (IF) returns after demodulation can be expressed as

$$
s_{r}(t)=B \cdot \exp \left(-j 2 \pi f_{i} \tau_{i}\right), \quad i=0,1, \ldots, N-1,
$$

where $B$ is the amplitude. Suppose the radial velocity of target is $\mathbf{v}_{\text {tgt }}$; then the round trip delay at $t=i T_{r}+T / 2+2 R / c$ is

$$
\begin{aligned}
\tau_{i} & =\frac{2\left(R-\left|\mathbf{v}_{\mathrm{tgt}}\right| t\right)}{c} \\
& =\frac{2 R}{c}-\frac{2\left|\mathbf{v}_{\mathrm{tgt}}\right|}{c}\left(i T_{r}+\frac{T_{p}}{2}+\frac{2 R}{c}\right) .
\end{aligned}
$$




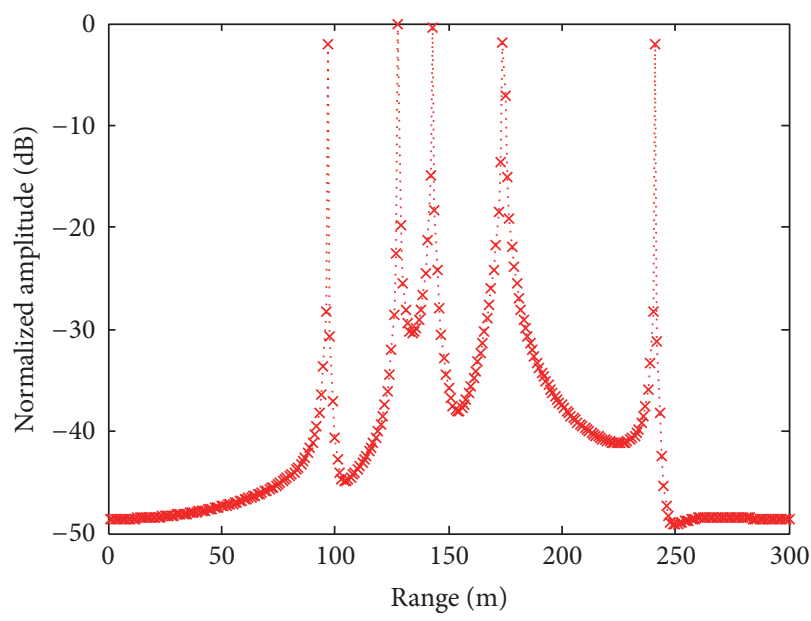

(a)

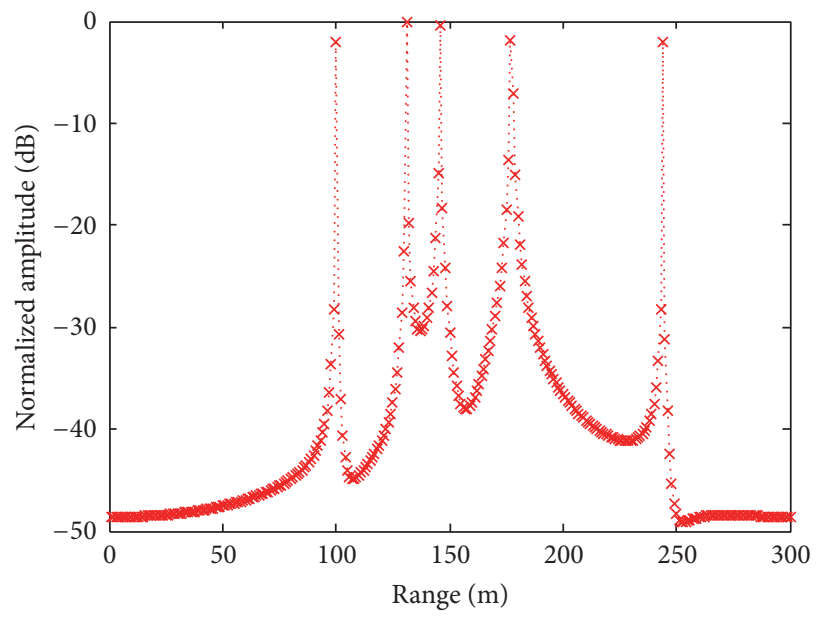

(c)

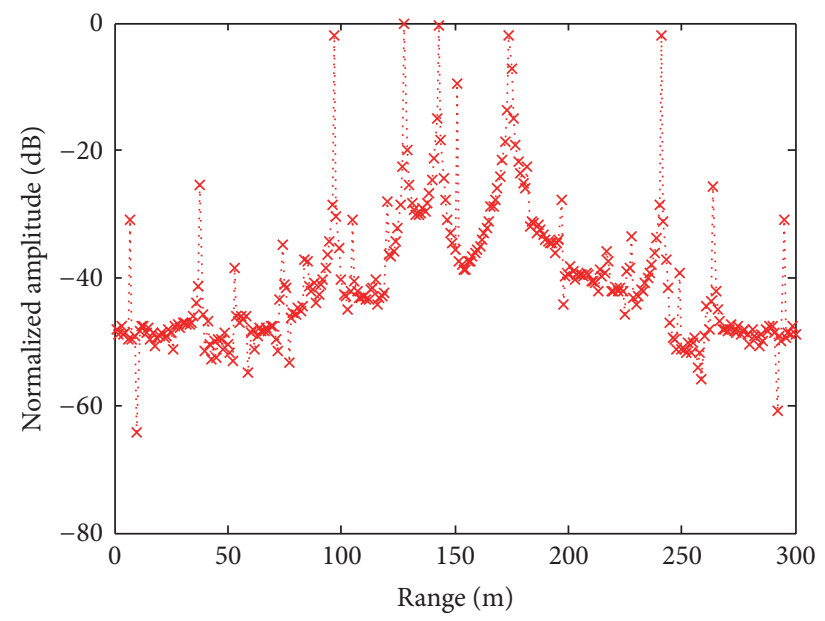

(b)

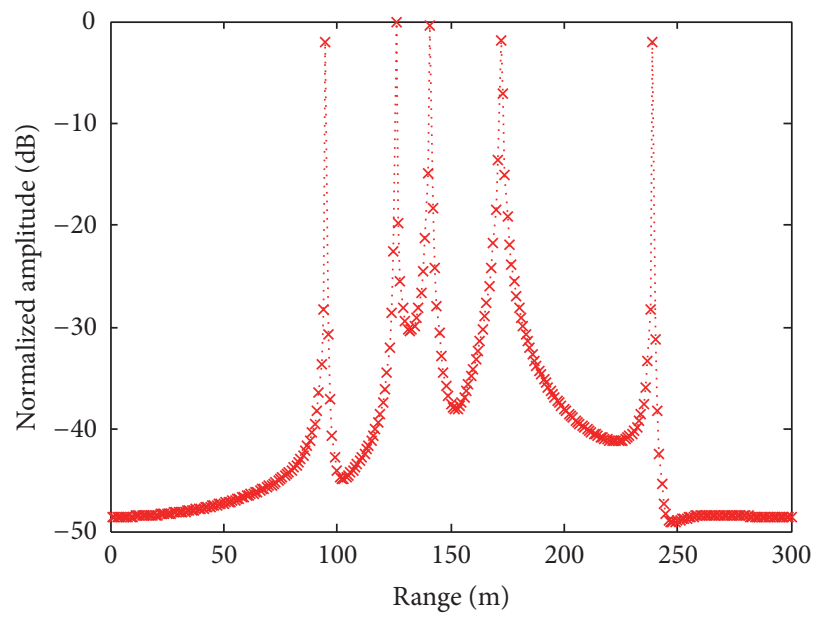

(d)

FIGURE 2: Illustration of the main drawbacks of traditional TDM for frequency stepped SAR.

According to (3) (7), the phase of $s_{r}(t)$ can be expressed as

$$
\begin{aligned}
\varphi_{i}= & -2 \pi i \Delta f \frac{2 R}{c}+2 \pi f_{0} \frac{2\left|\mathbf{v}_{\mathrm{tgt}}\right|}{c}\left(i T_{r}+\frac{T_{p}}{2}\right) \\
& +2 \pi i \Delta f \frac{2 R}{c} \frac{2\left|\mathbf{v}_{\mathrm{tgt}}\right|}{c}+2 \pi i \Delta f \frac{2\left|\mathbf{v}_{\mathrm{tgt}}\right|}{c}\left(i T_{r}+\frac{T_{p}}{2}\right) \\
& -2 \pi f_{0} \frac{2 R}{c}+2 \pi f_{0} \frac{2 R}{c} \frac{2\left|\mathbf{v}_{\mathrm{tgt}}\right|}{c} .
\end{aligned}
$$

All information of the target is contained in (8); taking IFFT of the return signal, range profile of the targets can be obtained.

\section{HRRP Profile Formation with Frequency Domain Extracted Method}

Conventional HRRP synthesis method is based on time domain inverse fast Fourier transform (IFFT) on data sequence sampled in each burst, which is denoted by TDM for short in the following. However, using conventional TDM to obtain HRRP has many disadvantages. Firstly, the quality of HRRP obtained by conventional TDM will deteriorate if the sampling time of each pulse is not accurately equal to $i T_{r}+T / 2+2 R / c$; secondly, conventional TDM cannot make good use of all return samples; it is not an effective method; thirdly, conventional TDM in time-domain use samples which contain noises and interferences, and there are no filtering operations in the processing; the obtained range profile is contaminated by undesired noise and interference. Due to the IFFT method itself, the precision of the range profile obtained by conventional TDM depends on the number of the pulses per burst adopted in radar system, which is not accurate enough. Figure 2 illustrated all the challenges which conventional TDM are faced with. Figure 2(a) is the result under the condition of accurate sampling time without interference, the profile is accurate in range positions, and all targets are clear, but due to the limited data point of the IFFT operation, which is equal to the number of the subpulses, the amplitudes of the range profile are not correct 


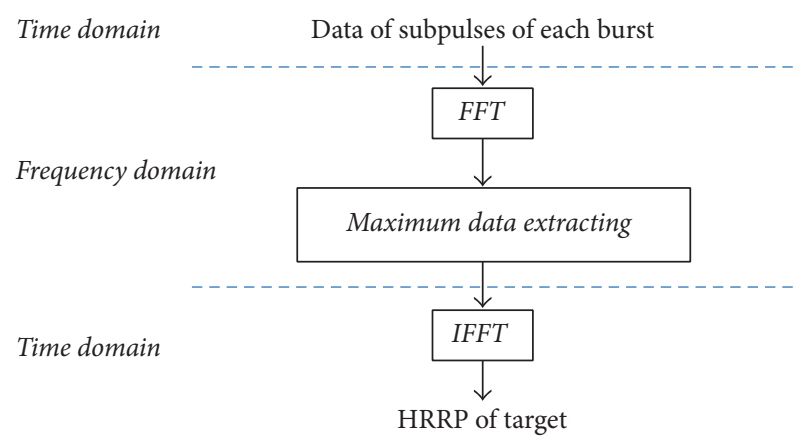

FIGURE 3: Basic idea and flowchart of the FDEM to obtain HRRP.

and all targets are set to be one in amplitude, but only one of the target's responses is correct; Figure 2(b) is the result under the condition of accurate sampling time but, with severe noise interference, the signal to noise ratio (SNR) is set to be $-20 \mathrm{~dB}$, and it is obvious that the effect of noise is fatal. There exist many artificial responses in the range profile which make it difficult to identify the real targets. Figure 2(c) is the result under inaccurate sampling time without noise interference. The sampling time is a bit earlier than the accurate sampling time, although the real targets can be clearly seen, and it is obvious that the range profile is changed and shifted compared to the result in Figure 2(a) due to the error of the sampling time, the amplitude of the profile are still incorrect; on the contrary, Figure 2(d) is the result under inaccurate sampling time without interference, but with the sampling time a bit later than the accurate sampling time. Although the real targets can be clearly seen, it is obvious that the phenomenon is similar to Figure 2(c), except for the range profile shifted to a contrary direction. All results prove that conventional TDM is unstable; its performance varies with the condition of radar system.

In order to overcome all challenges conventional TDM is faced with, a new frequency domain extracted method (FDEM) is proposed in this paper. The main idea and processing steps of this new method are described as follows.

There are three main steps to obtain a HRRP with FDEM.

Step 1. Take DFT of the sampled data of each subpulse, and obtain its frequency domain data firstly.

Step 2. Extract the maximum data in frequency domain of each subpulse and put them together sequentially to form a new frequency domain data.

Step 3. Taking IDFT of the frequency domain data, one can obtain the HRRP of the targets.

The basic processing idea and diagram of FDEM are shown in Figure 3. The FDEM has many advantages. Firstly, all useful returns of each subpulse can be received and used to synthesize the range profile, there is no need to select data samples, and, with such a processing method, the incorrect timing error which traditional IFFT method might face with can be avoided; secondly, the frequency domain extracting is applied, which act as a useful filter, only the data samples which have maximum SNR and contain the information of the target are extracted and used in the following IFFT processing, and the undesired negative impact of noise or interferences on HRRP are eliminated to some extent; thirdly, there is no quality deterioration caused by inaccurate sampling time and the amplitude of the range profile is stable and correct under any circumstance.

It is obvious that the FDEM can overcome all the challenges which conventional TDM is faced with. Its mathematical principle and theory formulas are described as follows.

Suppose the sampling interval is $T_{s}$ and the amplitude of the returned signal is normalized; the samples of the $i$ th return pulses can be expressed as

$$
\begin{aligned}
& s_{r}\left(n T_{s}\right) \\
& \quad=\exp \left(-j 2 \pi f_{0} \frac{2 R}{c}\right) \exp \left[j 2 \pi i \Delta f\left(n T_{s}-\frac{2 R}{c}\right)\right]
\end{aligned}
$$

in which $R$ is the range between target and radar, $c$ is the speed of light, and $\Delta f$ is the frequency increment. Denote (9) as

$$
\begin{aligned}
& s_{r}(n) \\
& \quad=\exp \left(-j 2 \pi f_{0} \frac{2 R}{c}\right) \exp \left[j 2 \pi i \Delta f\left(n T_{s}-\frac{2 R}{c}\right)\right] .
\end{aligned}
$$

The first phase term in (10) represents the phase delay of azimuth Doppler, and for simplicity of following deduces, it can be neglected before azimuth processing. Taking discrete Fourier transform (DFT) of each received pulse along range direction, the frequency domain signal can be expressed as

$$
\begin{aligned}
S_{r}(l) & =\sum_{n=0}^{N-1} s_{r}(n) \exp \left(-j \frac{2 \pi}{N} n \cdot l\right) \\
= & \sum_{n=0}^{N-1} \exp \left[j 2 \pi i \Delta f\left(n T_{s}-\frac{2 R}{c}\right)\right] \exp \left(-j \frac{2 \pi}{N} n \cdot l\right) \\
= & \exp \left(-j 2 \pi i \Delta f \frac{2 R}{c}\right) \\
& \cdot \sum_{n=0}^{N-1} \exp \left(j 2 \pi i \Delta f n T_{s}\right) \cdot \exp \left(-j \frac{2 \pi}{N} n \cdot l\right) \\
= & \exp \left(-j 2 \pi i \Delta f \frac{2 R}{c}\right) \\
& \cdot \sum_{n=0}^{N-1} \exp \left[j 2 \pi n\left(i \Delta f T_{s}-\frac{l}{N}\right)\right] \\
& =\exp \left(-j 2 \pi i \Delta f \frac{2 R}{c}\right) \frac{\sin \left[\pi N\left(i \Delta f T_{s}-l / N\right)\right]}{\sin \left[\pi\left(i \Delta f T_{s}-l / N\right)\right]} \\
& \cdot \exp \left[j \pi(N-1)\left(i \Delta f T_{s}-\frac{l}{N}\right)\right] .
\end{aligned}
$$

It is obvious that the maximum value of (11) can be achieved, if and only if $l=N i \Delta f T_{s}$. Extract the maximum 


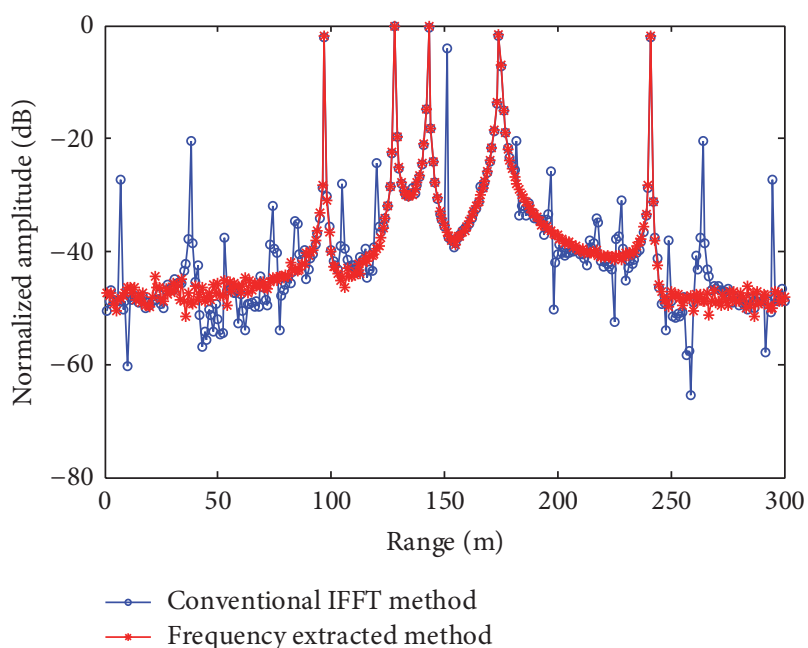

(a) Range profile comparison between FDE and conventional TDM

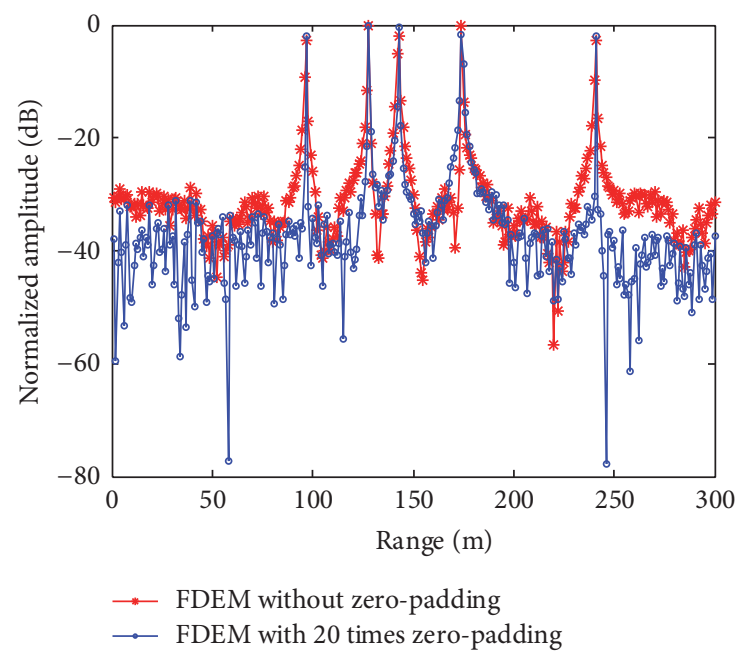

(b) Range profile comparison of FDEM with different zeropadding times

FIGURE 4: Range profile obtained by FDEM, SNR $=-20 \mathrm{~dB}$.

data of the frequency domain signals to form a new data sequence:

$$
s_{r t}(i)=N \exp \left(-j 2 \pi i \Delta f \frac{2 R}{c}\right) .
$$

Taking inverse discrete Fourier transform (IDFT) of the sequence expressed in (12), the range profile of the target with FDEM can be expressed as

$$
\begin{aligned}
& S_{r t}(r)=\frac{1}{N} \sum_{n=0}^{N-1} s_{r t}(n) \exp \left(j \frac{2 \pi}{N} n \cdot r\right) \\
&= \sum_{n=0}^{N-1} \exp \left(-j 2 \pi n \Delta f \frac{2 R}{c}\right) \exp \left(j \frac{2 \pi}{N} n \cdot r\right) \\
&= \frac{\sin [\pi N(r / N-\Delta f(2 R / c))]}{\sin [\pi(r / N-\Delta f(2 R / c))]} \\
& \cdot \exp [j \pi(N-1)(r / N-\Delta f(2 R / c))] .
\end{aligned}
$$

Obviously, the amplitude of the range profile can be expressed as:

$$
\left|S_{r t}(r)\right|=\left|\frac{\sin [\pi N(r / N-\Delta f(2 R / c))]}{\sin [\pi(r / N-\Delta f(2 R / c))]}\right| .
$$

Compared with the result of conventional TDM, the result of FDEM maintained the high resolution properties when noise interferences exist at the same time, and it can be effectively realized by fast Fourier transform (FFT). In fact, in the processing of the FDEM, by taking DFT of the received signal, the time domain signal is converted into frequencydomain, in which large volumes of noises are distributed on all frequency cells. While useful return signal of the targets is only concentrated and cumulated on specific frequency cells, the power density of noises is smaller than useful signals, and one can extract the useful data from ambient noises and eliminate the effect of the noise and interferences. It is obvious that FDEM has much better capability of antijamming than conventional TDM. Figure 4(a) illustrated a result of 5 targets with noise interferences, and the SNR is set to be $-20 \mathrm{~dB}$. It is obvious that using FDEM, the quality of the range profile could be easily controlled and maintained. In order to overcome the shortcoming of picket fence effect of FFT, one simplest way to improve the quality of range profile is zero-padding the data of return signal before taking DFT, with which the precision of frequency domain extraction in resolution, peak side lobe ratio (PSLR), integral side lobe ratio (ISLR), and the SNR loss of target can be greatly improved and guaranteed, as shown in Figure 4(b).

However, zero-padding is not an effective way to guarantee the quality of range profiles. There are more effective ways to overcome the shortcoming of picket fence effect of FFT, such as the chirp $z$ transform (CZT). The CZT algorithm is a digital signal processing algorithm which is applicable to the general case calculating limited width of the $z$-transform along the spiral curve. The CZT is more flexible than FFT in calculating the frequency spectrum; therefore it is used in various fields such as radar ranging and power prediction. Using the CZT algorithm one can efficiently evaluate the $z$ transform at $M_{z}$ points in the $z$-plane which lie on circular or spiral contours beginning at any arbitrary point in the $z$-plane. The angular spacing of the points is an arbitrary constant, and $M_{z}$ and $N$ are arbitrary integers.

The $z$-transform of a finite-number $N$ of nonzero points $x(n)$ is defined as

$$
X(z)=\sum_{n=0}^{N-1} x(n) z^{-n}
$$




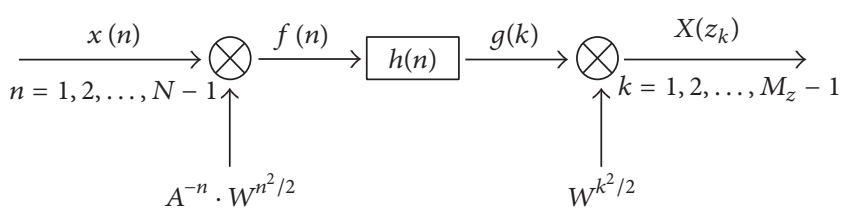

FIGURE 5: Illustration of steps involved in computing values of the $z$-transform using the CZT.

$$
\begin{aligned}
& \text { Let } \\
& \begin{aligned}
z_{k}=A \cdot W^{-k}=\frac{A_{0}}{W_{0}^{k}} \exp \left[j\left(k \varphi_{0}-\theta_{0}\right)\right] \\
\qquad k=0,1, \ldots, M_{z}-1, \\
X(k)=X\left(z_{k}\right)=\sum_{n=0}^{N-1} x(n) A^{-n} W^{n k}, \\
k=0,1, \ldots, M_{z}-1,
\end{aligned}
\end{aligned}
$$

where both $A$ and $W$ are arbitrary complex points of the form:

$$
\begin{gathered}
A=A_{0} \exp \left(-j \theta_{0}\right), \\
W=W_{0} \exp \left(-j \varphi_{0}\right) .
\end{gathered}
$$

Using the ingenious substitution, there exists

$$
n k=\frac{n^{2}+k^{2}-(k-n)^{2}}{2} .
$$

Then an expression is produced:

$$
\begin{array}{r}
X(k)=X\left(z_{k}\right)=\sum_{n=0}^{N-1} x(n) A^{-n} W^{n^{2} / 2} W^{k^{2} / 2} W^{-(k-n)^{2} / 2}, \\
k=0,1, \ldots, M_{z}-1 .
\end{array}
$$
to

New sequences $f(n), h(n), g(k)$ will be formed according

$$
\begin{aligned}
& f(n)=x(n) A^{-n} W^{n^{2} / 2}, \quad n=0,1,2, \ldots, N_{z}-1, \\
& h(n)=W^{-n^{2} / 2}, \quad n=0,1,2, \ldots, N_{z}-1, \\
& g(k)=\sum_{n=0}^{N_{z}-1} f(n) h(k-n), \quad k=0,1, \ldots, M_{z}-1 .
\end{aligned}
$$

Then, the CZT of $x(n)$ can be realized as

$$
X(k)=g(k) W^{k^{2} / 2}, \quad k=0,1, \ldots, M_{z}-1 .
$$

The operation of the whole process can be illustrated as Figure 5.

Because the DFT of $x(n)$ can be seen as the $z$ transform of $x(n)$ along the unit sphere circle on the $z$ plane, it can be realized through CZT which has the flexibility of defining the output spectrum region. This flexibility can be used to analyze the most important region of the spectrum, other than the whole region traditional DFT does. The output point number of the most important local spectrum can be defined arbitrarily, which means the output of the CZT can be a zoomed version of the local spectrum, with which the maximum data can be extracted more accurately than DFT. This advantage can greatly enhance the precision of range profile. Thus, CZT transforms can be adopted in FDEM to substitute the FFT operation before frequency domain data extracting process, which is illustrated in Figure 6(a).

CZT has the flexibility for computing the local spectrum around the maximum frequency data which plays the most important role in HRRP obtaining of frequency stepped radar, and the precision of operation can be greatly guaranteed and the efficiency of FDEM can be greatly improved. However, note the fact that most useful signals of frequency stepped synthetic aperture radar always have the same frequencies as the transmitted signal does (mostly the stationary scene, except moving targets, which must be considered separately with GMTI techniques); therefore the most important data which contains the meaningful information of the scene in frequency domain should be located at the same frequency cell. This simple fact paves a direct way to realize the FDEM through the computing of the most important data in frequency, which is located at the frequency cell with the same frequency as the transmitted signal does. According to the theory of DFT, the frequency domain value of a point located at the frequency $f_{i}$ can be directly computed with

$$
v_{f_{i}}=\sum_{n=0}^{N-1} x(n) \exp \left(-j 2 \pi f_{i} n T_{s}\right),
$$

with which the FDEM can be further simplified as single point Fourier transform (SPFT) operations followed by an IFFT operation without maximum data searching and extracting as Figure 6(b) depicted, and the range profiles $S_{r t}(r)$ of the target can be obtained by IFFT processing:

$$
\begin{aligned}
S_{r t}(r)=\frac{1}{N} \sum_{n=0}^{N-1} v_{f_{i}}(i) \exp \left(j \frac{2 \pi}{N} n \cdot r\right), & \\
r & =0,1,2, \ldots, N-1 .
\end{aligned}
$$

In order to validate the methods proposed above, experiments with both virtual and real radar data under different noise level are executed, and all results are illustrated in Figure 7.

It can be seen from Figures 7(a) and 7(b) that the antinoise ability of FDEM is good enough. Correct and clear range profile of the target can be guaranteed under different noise level, and the responses of the scattering centers are stable and robust except the side lobe level interfered with noise. Furthermore, the real data processing results in Figures 7 (c) and $7(\mathrm{~d})$ prove that the result of FDEM is much better than TDM ones, which has severe ghost peaks in the range profile. It is clearly incorrect and should be carefully eliminated before synthetic aperture imaging processing, or else it will lead to several ghost response in the final SAR images. 


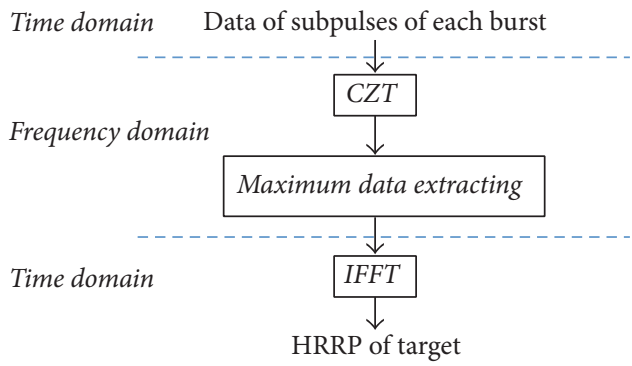

(a) FDEM with CZT

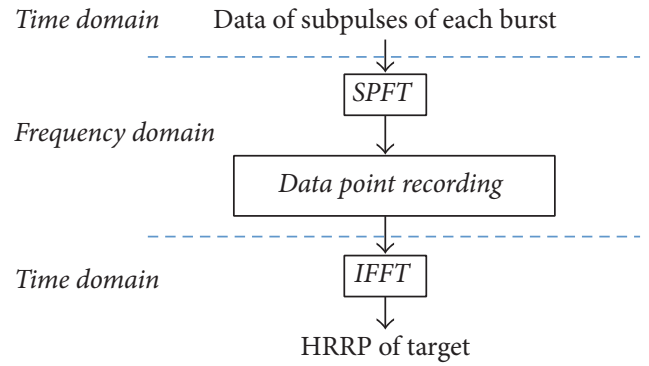

(b) FDEM with SPFT

FIGURE 6: FDEM with CZT and SPFT.
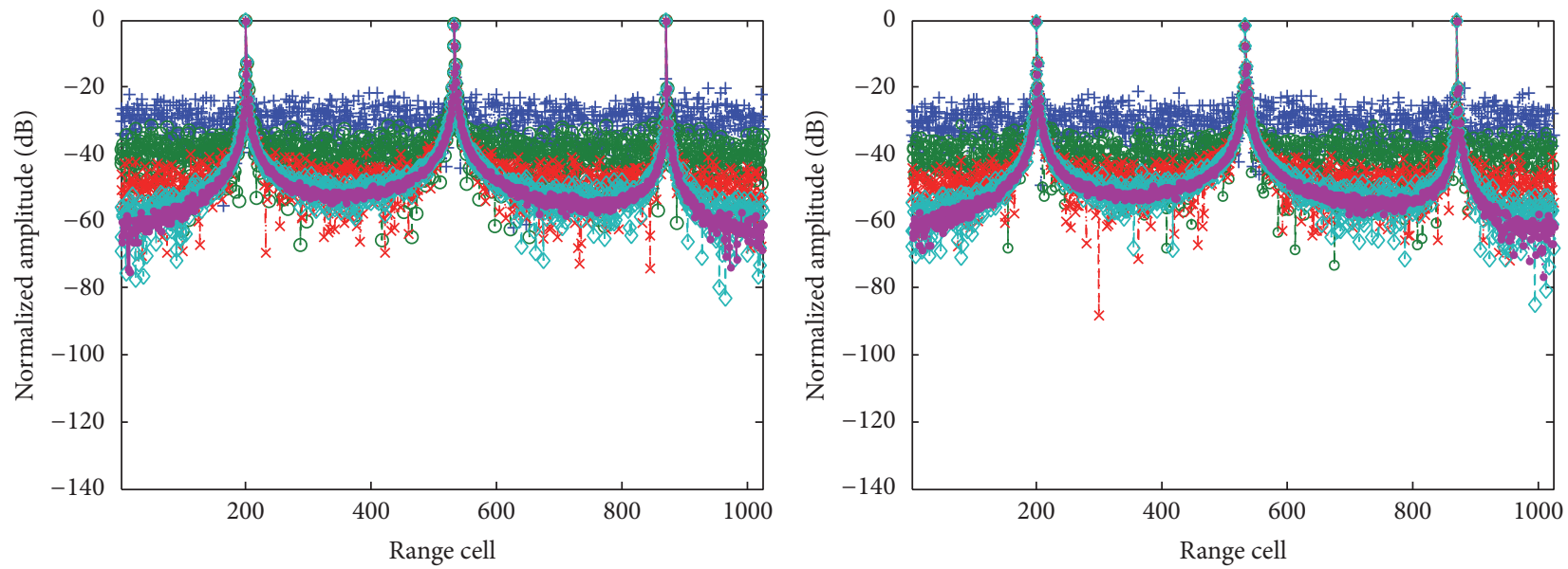

$$
\begin{aligned}
-\cdots S J R & =-30 \mathrm{~dB} \\
-\ominus-\mathrm{SJR} & =-20 \mathrm{~dB} \\
-\times-\mathrm{SJR} & =-10 \mathrm{~dB}
\end{aligned}
$$

(a) HRRP of FDEM with CZT

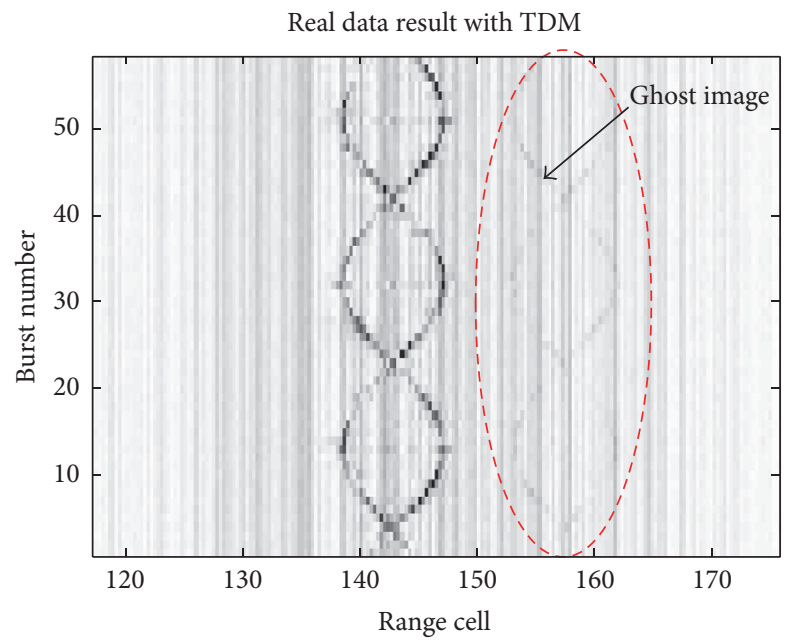

(c) Real Data HRRP with TDM

$$
\begin{aligned}
& \text { +. } \mathrm{SJR}=-30 \mathrm{~dB} \\
& -\Theta-\mathrm{SJR}=-20 \mathrm{~dB} \\
& -x-\mathrm{SJR}=-10 \mathrm{~dB} \\
& -\diamond-S J R=0 d B \\
& \text {-. S SRR }=10 \mathrm{~dB}
\end{aligned}
$$

(b) HRRP of FDEM with SPFT

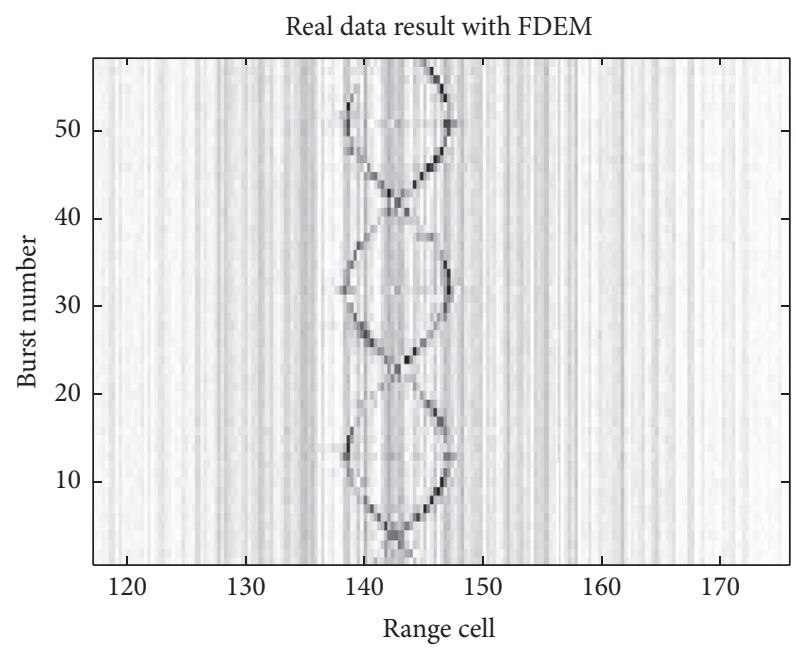

(d) Real Data HRRP with FDEM

FIGURE 7: Virtual Data HRRP obtained by FDEM with CZT and SPFT plus real data results comparison with conventional TDM and proposed FDEM. 


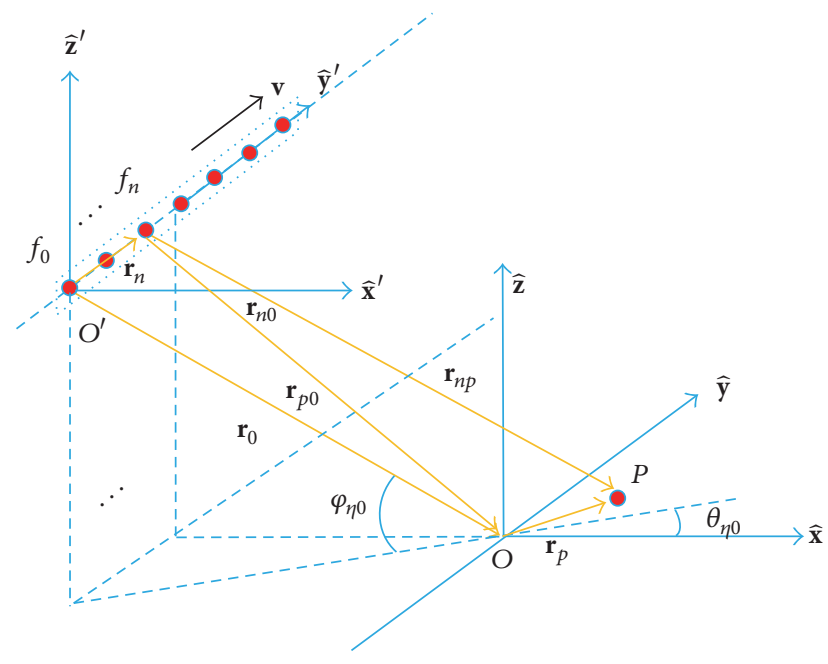

FIGURE 8: Imaging geometry of the frequency stepped SAR.

Note the fact that no matter how the frequency extracted data is obtained, the range profile of a point target can only obtained if and only if at $r=N \Delta f \cdot 2 R / c$, according to (13) and (14). For azimuth imaging processing, classical Range-Doppler algorithm (RDA), range migration algorithm (RMA), polar format algorithm (PFA), and so on cannot be directly used; they must be modified to fit the specific characteristic of the range profile obtained by FDEM. This is the significant difference between frequency stepped SAR imaging and traditional LFM based SAR. In order to realize azimuth processing for focused images of the interested scene, back projection algorithm (BPA) is the simplest and direct way capable for azimuth imaging.

However, before initialization of the azimuth processing, there still is some work to do. Note the formulas deduced all above; there are some important phase errors which have been neglected altogether. However, it is not true in real imaging applications. Because the radar platform is moving during each pulse burst, which lead to a different round trip delay for the same target within each cycle of the subpulse, and the frequency domain extracted data of each subpulse will have different phase even for the same target, this is awful for the imaging processing and the phase difference between each subpulse must be compensated firstly. Suppose the moving velocity of the radar platform is $\mathbf{v}$; the subpulse interval is $T_{r}$; the radar system adopts $N$ subpulses per burst. It is obvious that the spacing distance of the radar platform moving forward during adjacent subpulse interval is $d=|\mathbf{v}| T_{r}$, as it is moving forward. Subpulses with sequential frequency coding are transmitted and received by the antenna, as depicted in Figure 8.

The vector $\mathbf{r}_{n p}$ can be combined to form $\mathbf{r}_{n 0}$ from the $n$th position of the antenna to the scene center. After inverting the direction of $\mathbf{r}_{n}$ to point from the $n$th position of the antenna to the reference ones, $\mathbf{r}_{n 0}$ can be expressed as

$$
\mathbf{r}_{n 0}=\mathbf{r}_{0}-\mathbf{r}_{n}
$$

Finally, $\mathbf{r}_{n p}$ describes the location of point $p$ with respect to the $n$th position of antenna which can be expressed as

$$
\mathbf{r}_{n p}=\mathbf{r}_{n 0}+\mathbf{r}_{p}=\mathbf{r}_{0}-\mathbf{r}_{n}+\mathbf{r}_{p} .
$$

In general, the normalized transmitted radar signal from the $n$th subpulse is expressed as

$$
s_{n}(t)=\exp \left(j 2 \pi f_{n} t\right),
$$

where $f_{n}$ is the operational frequency, and the initial phase is traditionally assumed to be zero for convenience. After transmission, the signal propagates in the space and is finally scattered by the target and then received by the antenna, and the echo can be expressed as

$$
s_{n}(t, \mathbf{k})=\exp \left[j 2 \pi f_{n} t-2 \mathbf{k}_{n} \cdot \mathbf{r}_{n p}\right],
$$

where $\mathbf{k}_{n}$ is the wavenumber and denotes the radar line-ofsight to the reference path, and it can be expressed as

$$
\mathbf{k}_{n}=k_{n} \widehat{\mathbf{r}}_{n 0}
$$

and the magnitude of this vector satisfies

$$
k_{n}=\frac{2 \pi f_{n}}{c} .
$$

In traditional theory, the far-field approximation assumes

$$
\mathbf{k}_{n}=k_{n} \widehat{\mathbf{r}}_{0},
$$

where $\widehat{\mathbf{r}}_{0}$ denotes the unit vector of $\mathbf{r}_{0}$. With the above approximations the received signal from point $p$ due to the $n$th subpulse can be expressed as

$$
\begin{aligned}
& s_{n}\left(t, \mathbf{r}_{p}, \widehat{\mathbf{r}}_{0}\right) \\
& \quad=\exp \left\{j\left[2 \pi f_{n}\left(t-t_{0}\right)+2 \mathbf{k}_{n} \cdot \mathbf{r}_{n}-2 \mathbf{k}_{n} \cdot \mathbf{r}_{p}\right]\right\},
\end{aligned}
$$

in which $t_{0}=2\left|\mathbf{r}_{0}\right| / c$. Figure 8 illustrates the following:

$$
\begin{aligned}
k_{x} & =\cos \varphi_{\eta 0} \cos \theta_{\eta 0}, \\
k_{y} & =\cos \varphi_{\eta 0} \sin \theta_{\eta 0}, \\
k_{z} & =\sin \varphi_{\eta 0}, \\
\widehat{\mathbf{r}}_{0} & =k_{x} \widehat{\mathbf{x}}+k_{y} \widehat{\mathbf{y}}+k_{z} \widehat{\mathbf{z}}, \\
\mathbf{r}_{n} & =\left[0, y_{n} \widehat{\mathbf{y}}, 0\right]=[0, n d \cdot \widehat{\mathbf{y}}, 0], \\
n & \quad n=0,1,2, \ldots, N-1,
\end{aligned}
$$

where $d=|\mathbf{v}| \cdot P R T_{\text {sub }}$ is the distance between two adjacent positions and $N$ is the subpulse number, which is supposed to be odd in this paper. Suppose the operational frequency of the $n$th subpulse satisfies

$$
f_{n}=f_{0}+n \Delta f, \quad n=0,1,2, \ldots, N-1
$$




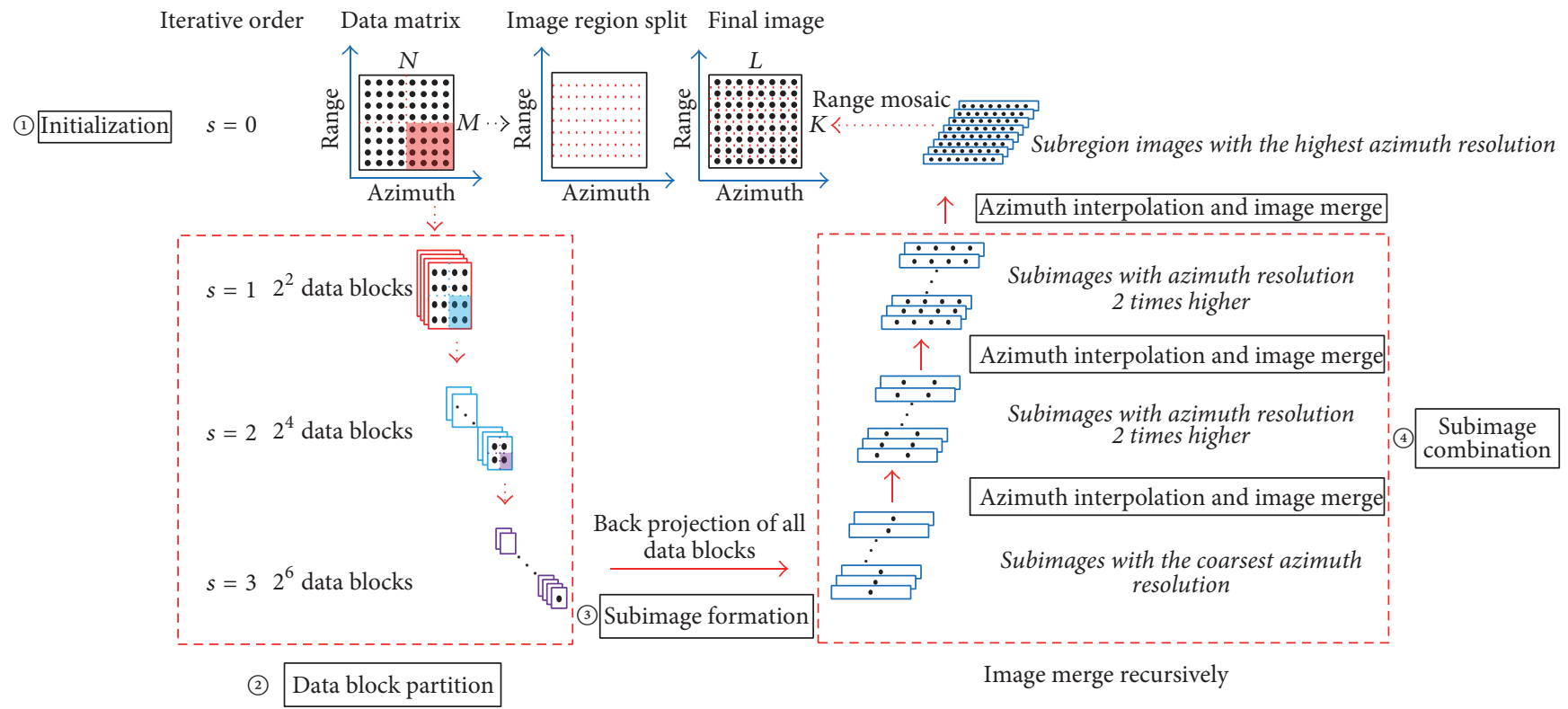

FIGURE 9: Schematic diagram of DBP-FFBPA for frequency stepped SAR imaging.

in which $\Delta f=1 / T_{p}$ and $T_{p}$ is the pulse width. Suppose the reflected factor of the target is $\sigma$; by applying (34), (35) in (33), the received echo can be expressed as

$$
\begin{aligned}
& s\left(t, \mathbf{r}_{p}, \widehat{\mathbf{r}}_{0}\right)=\sigma \exp \left[j 2 \pi f_{n}\left(t-t_{0}-\frac{2 \widehat{\mathbf{r}}_{0} \cdot \mathbf{r}_{p}}{c}\right)\right] \\
& \cdot \exp \left\{j\left[2 n k_{0} d k_{y}+2 n^{2} \Delta k d k_{y}\right]\right\}=\sigma \\
& \cdot \exp \left[j 2 \pi f_{n}\left(t-\frac{2\left|\mathbf{r}_{p 0}\right|}{c}\right)\right] \\
& \cdot \exp \left\{j \left[\frac{4 \pi f_{0}}{c} n d \cos \varphi_{\eta 0} \sin \theta_{\eta 0}\right.\right. \\
& \left.\left.+\frac{4 \pi \Delta f}{c} n^{2} d \cos \varphi_{\eta 0} \sin \theta_{\eta 0}\right]\right\},
\end{aligned}
$$

in which $\mathbf{r}_{p 0}$ is the range vector between radar and target at the first cycle of subpulses in each burst. It is obvious that the second phase term in (22) must be compensated before applying FDEM; the compensation phase factor is

$$
\begin{gathered}
\mathrm{Pha}_{c}=\exp \left\{-j\left[\frac{4 \pi f_{0}}{c} n d \cos \varphi_{\eta 0} \sin \theta_{\eta 0}\right.\right. \\
\left.\left.+\frac{4 \pi \Delta f}{c} n^{2} d \cos \varphi_{\eta 0} \sin \theta_{\eta 0}\right]\right\} .
\end{gathered}
$$

After phase compensation, the echoes of each subpulse can be used for range profile extracting through FDEM, and then the imaging processing of frequency stepped SAR can be executed.

The application of BPA in engineering practice is greatly restricted by its huge computational amount. In order to improve its computational efficiency, a series of fast BPA is proposed in [24-27]. As a typical representative of fast BPA, fast factorized BPA in [28-36] accelerate the computation speed of BPA through the idea of progressive iterative accumulation and can get a speedup comparable to frequency domain algorithm. The ideas in $[37,38]$ have paved the way to an enhanced fast factorized back projection algorithm based on data block partition. The new algorithm is designed, modified, and adopted in this paper for the imaging of frequency stepped SAR.

According to SAR imaging theory, image resolution is inversely proportional to SAR azimuth steering angle. The larger the azimuth steering angle is, the higher the image resolution is. Furthermore, if the length of data matrix $s_{r}(m, n)$ was shortened into $1 / N$ times compared to the original size in range direction, no matter which part of the scene will be imaged, the size of imaging region in range direction will be shortened to approximately $1 / N$ of the whole range region, which means there is only $1 / N$ effective range grid which need to be back projected; meanwhile, if the length of data matrix $s_{r}(m, n)$ was shortened into $1 / N$ times compared to the original size in azimuth direction, the image resolution will be lower by a factor of $1 / N$ correspondingly due to the narrower range of azimuth steering angle. Therefore, if we shorten both directions of data matrix, the image grids which must be back projection will be lesser by a factor of $1 / N^{2}$. Assume $I \in C^{K \times L}$ is the final image matrix and $\mathbf{p}_{k l}=\left(p_{k l}^{x}, p_{k l}^{y}, p_{k l}^{z}\right)^{T}$ is the coordinates of the final image grids, which satisfy $p_{k l}^{x}=k \rho_{r}, p_{k l}^{y}=l \rho_{a}, k=1,2, \ldots, K$, $l=1,2, \ldots, L$. Figure 9 shows the schematic diagram of the DBP-FFBPA, where data block partition factor $q$ equals 2 and the iterative order is 3 .

Based on all theories deduced above, the imaging processing of the frequency stepped SAR imaging can be realized as follows. 
Step 1. Phase compensation for all subpulses according to (36) and (37) is executed. Then acquire the range profile data based on FDEM. According to the formulas deduced in Section 2, the range profile data can be obtained through frequency extracted data of all subpulses in the same burst; in order to obtain precision result of the range profiles, CZT or SPFT can be adopted alternatively, after that, a data matrix of range profile $s_{r}(m, n)$ of thousands of burst can be obtained for azimuth imaging processing.

Step 2. Data block partition of data matrix $s_{r}(m, n)$ is executed. Firstly, determine the value of data block partition factor $q$ and iterative order $s$ according to the specific imaging requirements. Secondly, calculate subdata matrix $\widehat{\mathbf{s}}_{\text {block }}\left(m^{\prime}, n^{\prime}\right)$ and the SAR position vector $\mathbf{p}_{t}^{\prime}$ corresponding to each subdata matrix. The expressions are as follows:

$$
\begin{aligned}
\widehat{\mathbf{s}}_{\text {block }}\left(m^{\prime}, n^{\prime}\right) & =s_{r}\left(a \frac{M}{q^{s}}+m^{\prime}, b \frac{N}{q^{s}}+n^{\prime}\right), \\
\mathbf{p}_{t}^{\prime}\left(n^{\prime}\right) & =\mathbf{p}_{t}\left(b \frac{N}{q^{s}}+n^{\prime}\right)
\end{aligned}
$$

in which $a=0: q^{s}-1, b=0: q^{s}-1, m^{\prime}=1: M / q^{s}$, and $n^{\prime}=$ $1: N / q^{s}$. At the same time, the coordinates of corresponding subimage grids are $\mathbf{p}_{k^{\prime} l^{\prime}}^{\prime}=\left(p_{k^{\prime} l^{\prime}}^{\prime x}, p_{k^{\prime} l^{\prime}}^{\prime y}, p_{k^{\prime} l^{\prime}}^{\prime z}\right)^{T}$, where $p_{k^{\prime} l^{\prime}}^{\prime x}=$ $k^{\prime} \cdot \rho_{r}, p_{k^{\prime} l^{\prime}}^{\prime y}=l^{\prime} \cdot q^{s} \rho_{a}, k^{\prime}=1: K / q^{s}$, and $l^{\prime}=1: L / q^{s}$.

Step 3. After data block partition operations, there are $q^{2 s}$ subdata matrixes $\left\{\widehat{\boldsymbol{s}}_{\text {block }}\right\}_{q^{2 s}}$ and for each subdata matrix, subimage formation is executed by back projection. It is important to note that the unambiguous range of frequency stepped SAR is

$$
R_{u}=\frac{c T_{p}}{2}=\frac{c / 2}{\Delta f} .
$$

According to (13) and (14), if and only if

$$
r=\frac{N \Delta f \cdot 2 R}{c}
$$

the range profile of a point target with range $R$ can only be achieved. Due to the finite point of IFFT, the range profile of a point target with range $R$ which is bigger than the unambiguous range $R_{u}$ can only be achieved at

$$
r=\frac{2 N \Delta f \cdot \operatorname{rem}\left(R, R_{u}\right)}{c},
$$

where $\operatorname{rem}\left(R, R_{u}\right)$ denotes the remainder operation.

After processing every subdata matrix $\left\{\widehat{\mathbf{s}}_{\text {block }}\right\}_{q^{2 s}}$ with back projecting operation, a set of subimages $\left\{I_{\text {block }}\left(k^{\prime}, l^{\prime}\right)\right\}_{q^{2 s}}$ with the coarsest azimuth resolution are obtained.

Step 4. Subimage combination is executed and the final imaging results are output. During the process of subimage combination, DBP-FFBPA merges with the subimages to generate the final image. Firstly, azimuth image merge is
TABLE 1: Simulation parameters.

\begin{tabular}{lc}
\hline Simulation parameters & Values \\
\hline Reference carrier frequency $(\mathrm{GHz})$ & 3 \\
Number of subpulse per burst & 301 \\
Frequency step $(\mathrm{MHz})$ & 0.5 \\
Pulse width (us) & 2 \\
Azimuth beam width $\left(^{\circ}\right)$ & 1.91 \\
Platform height $(\mathrm{km})$ & 8 \\
Platform velocity $(\mathrm{m} / \mathrm{s})$ & 150 \\
Reference slant range $(\mathrm{km})$ & 11 \\
Sampling frequency $(\mathrm{MHz})$ & 15 \\
Repetition frequency of burst $(\mathrm{Hz})$ & 831 \\
Repetition frequency of subpulse $(\mathrm{KHz})$ & 250 \\
Scene size-range $(\mathrm{m}) \times$ azimuth $(\mathrm{m})$ & $300 \times 300$ \\
Target scene & $3 \times 3$ Point array \\
Ground resolution-range $(\mathrm{m}) \times$ azimuth $(\mathrm{m})$ & $1.5 \times 1.5$ \\
\hline
\end{tabular}

executed recursively to form a series of new subimages with improved azimuth resolution 2 times higher in azimuth direction each cycle during image merge process within the same range region; then new subimages of different range region are merged together through mosaic method to form the final image. The newly merged subimages within different range region can be generated recursively using the subimages corresponding to each data block or each merge cycle. It should be pointed out that the grid points of the new subimage, which are obtained by the combination of former subimages, are twice the size of the former subimage grid points in azimuth direction and their azimuth resolution is 2 times higher than that of the former subimages. Due to the fact that SAR image is a complex image, interpolation should be operated on both phase and amplitude of the subimages during image combinations. In this paper, the interpolation methods of phase and amplitude are selected as "nearest" and "spline," respectively.

\section{Imaging Validations}

In order to validate the practicability of the proposed FDEM and imaging algorithm, simulations are executed and the point spread function of frequency stepped SAR is obtained. The simulation parameters are given in Table 1 . The imaging result of a uniformly spacing point array of 9 point targets is illustrated in Figure 10 and their corresponding 3D point spread functions are depicted in Figure 11. The numerical side lobe properties of point spread function are collected in Table 2. It is apparent that the imaging result is perfect, all targets in the scene are well focused, and the properties of point spread function are nearly the same as theory properties. The imaging algorithm based on FDEM and DBPFFBPA is not only correct but also effective.

\section{Conclusion}

With frequency stepped waveform, high resolution range profile of the targets can be obtained, and by using frequency 

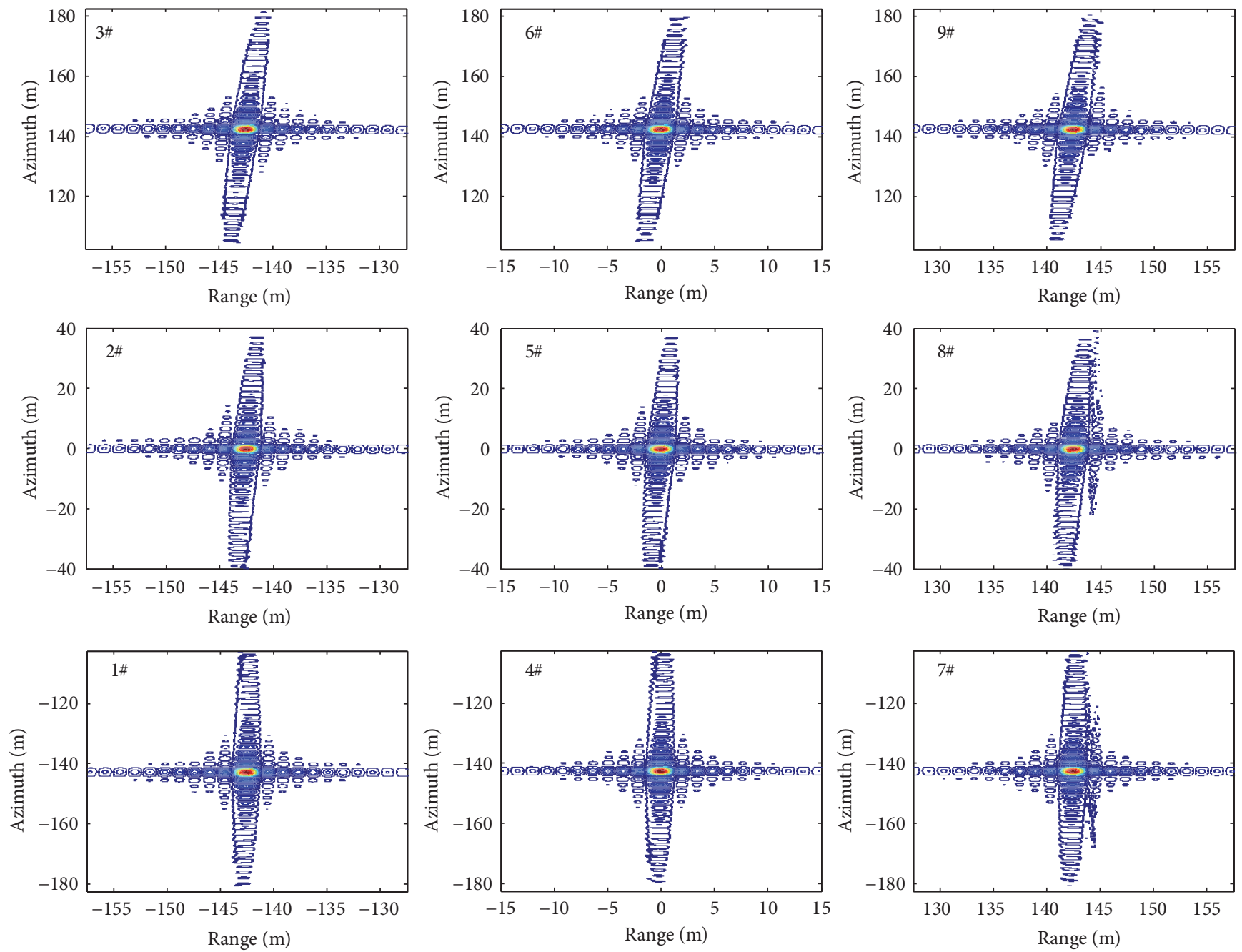

FIGURE 10: Imaging result of frequency stepped SAR with FDEM and DBP-FFBPA, dynamic range: $40 \mathrm{~dB}$.

TABLE 2: Side lobe property of PSF.

\begin{tabular}{lcc}
\hline Target number & PSLR $(\mathrm{dB})$ & ISLR $(\mathrm{dB})$ \\
\hline 1 & -13.2103 & -10.9574 \\
2 & -13.1929 & -11.0083 \\
3 & -13.2146 & -11.0712 \\
4 & -13.2103 & -10.9664 \\
5 & -13.1876 & -11.0090 \\
6 & -13.2000 & -11.0122 \\
7 & -13.2103 & -10.9565 \\
8 & -13.1903 & -11.0190 \\
9 & -13.1929 & -11.0102 \\
\hline
\end{tabular}

stepped waveform in synthetic aperture radar, high crossrange resolution can also be obtained; thus high resolution images of target area can be obtained. In this paper, a novel frequency domain extracted method (FDEM) to obtain high range resolution profile (HRRP) for frequency stepped synthetic aperture radar (SAR) is proposed, and the mathematical principle and formulas of this new HRRP obtaining idea combined with classical fast Fourier transform (FFT), single point Fourier transform (SPFT), and chirp $z$ transform (CZT) are deduced, analyzed, and compared in detail. Based on the HRRP data, precision imaging processing was completed using a data block partition based fast factorized back projection algorithm. Imaging validations are executed and the results are given in the end. All results proved that the FDEM has great capability of antijamming. It is more effective than conventional IFFT method and shows a great promise in frequency stepped radar imaging and applications.

\section{Competing Interests}

The authors declare that they have no competing interests. 

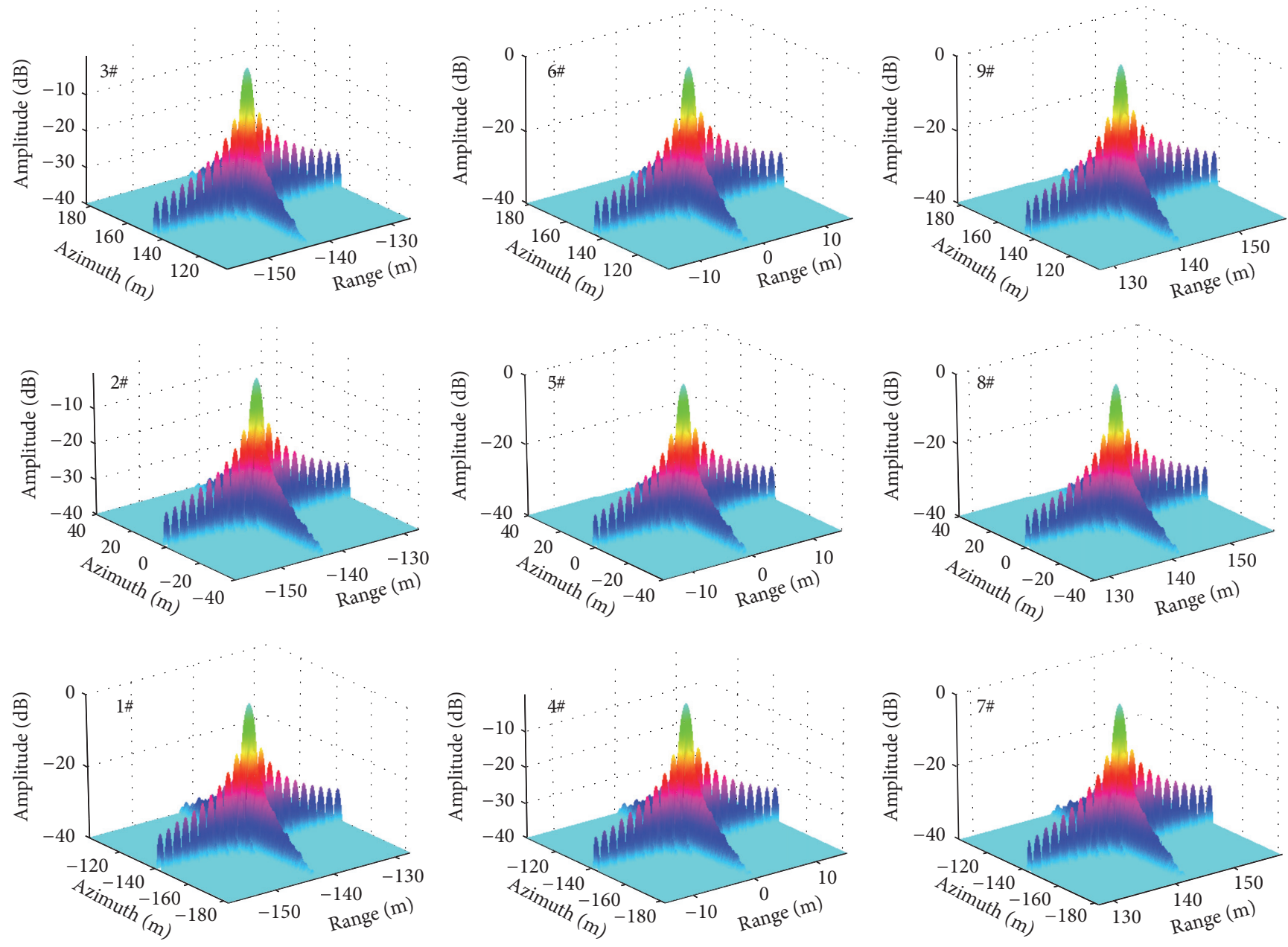

FIGURE 11: Point spread function of frequency stepped SAR with FDEM and DBP-FFBPA, dynamic range: $40 \mathrm{~dB}$.

\section{References}

[1] T. Long, E. Mao, and Y. Han, "High range resolution performance of frequency stepped radar signal," in Proceedings of the CIE International Conference of Radar Proceedings (ICR '96), pp. 242-245, Beijing, China, October 1996.

[2] I. Gladkova, "Analysis of stepped-frequency pulse train design," IEEE Transactions on Aerospace and Electronic Systems, vol. 45, no. 4, pp. 1251-1261, 2009.

[3] S. M. Wu, G. A. Ybarra, and W. E. Alexander, "A complex optimal signal-processing algorithm for frequency-stepped CW data," IEEE Transactions on Circuits and Systems II: Analog and Digital Signal Processing, vol. 45, no. 6, pp. 754-757, 1998.

[4] H. Schimpf, A. Wahlen, and H. Essen, "High range resolution by means of synthetic bandwidth generated by frequency-stepped chirps," Electronics Letters, vol. 39, no. 18, pp. 1346-1348, 2003.

[5] T. Zeng, P. Yin, and Q. Liu, "Wideband distributed coherent aperture radar based on stepped frequency signal: theory and experimental results," IET Radar, Sonar and Navigation, vol. 10, no. 4, pp. 672-688, 2016.

[6] G. A. Ybarra, G. L. Bilbro, S. M. Wu, and S. H. Ardalan, "High resolution target range estimation using frequency-stepped CW radar," in Proceedings of the IEEE MTT-S International
Microwave Symposium Digest, vol. 1, pp. 249-252, Atlanta, Ga, USA, 1993.

[7] G. A. Ybarra, S. H. Ardalan, C. P. Hearn, R. E. Marshall, and R. T. Neece, "Detection of target distance in the presence of an interfering reflection using a frequency-stepped double side-band suppressed carrier microwave radar system," IEEE Transactions on Microwave Theory and Techniques, vol. 39, no. 5, pp. 809-818, 1991.

[8] H. Wang, V. Dang, L. Ren et al., "An elegant solution: an alternative ultra-wideband transceiver based on stepped-frequency continuous-wave operation and compressive sensing," IEEE Microwave Magazine, vol. 17, no. 7, pp. 53-63, 2016.

[9] H. J. Yuan, M. G. Gao, and G. M. Liu, "Coherent spectrum synthesis of frequency-stepped chirp signal," in Proceedings of the IET International Radar Conference, pp. 1-4, Guilin, China, 2009.

[10] H. F. Wang, L. Y. Ren, E. Mao, and A. E. Fathy, "Phase based motion characteristics measurement for fall detection by using stepped-frequency continuous wave radar," in Proceedings of the IEEE Topical Conference on Biomedical Wireless Technologies, Networks, and Sensing Systems (BioWireleSS '16), pp. 43-45, Austin, Tex, USA, 2016.

[11] P. Lindelöw and J. J. Mohr, "Coherent lidar modulated with frequency stepped pulse trains for unambiguous high duty cycle 
range and velocity sensing in the atmosphere," in Proceedings of the IEEE International Geoscience and Remote Sensing Symposium (IGARSS '07), pp. 2787-2790, Barcelona, Spain, June 2007.

[12] F. Zhu, Q. Zhang, Q. Lei, and Y. Luo, "Reconstruction of moving target's HRRP using sparse frequency-stepped chirp signal," IEEE Sensors Journal, vol. 11, no. 10, pp. 2327-2334, 2011.

[13] H. Qiongdan, L. Yong, C. Wei, L. Weihua, and L. Bo, "A new multicarrier chaotic phase coded stepped-frequency pulse train radar signal and its characteristic analysis," in Proceedings of the 10th IEEE Conference on Industrial Electronics and Applications (ICIEA '15), pp. 444-448, IEEE, Auckland, New Zealand, June 2015.

[14] H. Liu, H. Jiang, X. Yang, and J. Wang, "Amplitude and phase calibration of digital array radar using frequency stepped signals," in Proceedings of the IET International Radar Conference, pp. 1-5, Shanxi, China, April 2013.

[15] G. Xia, H. Su, and P. Huang, "Velocity compensation methods for LPRF modulated frequency stepped-frequency (MFSF) radar," Journal of Systems Engineering and Electronics, vol. 21, no. 5, pp. 746-751, 2010.

[16] Kusmadi and A. Munir, "Simulation design of compact steppedfrequency continuous-wave through-wall radar," in Proceedings of the 5th International Conference on Electrical Engineering and Informatics (ICEEI '15), pp. 332-335, Denpasar, Indonesia, August 2015.

[17] K. Huo, B. Deng, Y. Liu, W. Jiang, and J. Mao, “The principle of synthesizing HRRP based on a new OFDM phase-coded stepped-frequency radar signal," in Proceedings of the IEEE 10th International Conference on Signal Processing (ICSP '10), pp. 1994-1998, IEEE, Beijing, China, October 2010.

[18] S. Xin, J. Ge, and B. Wu, "Research on high resolution SAR based on frequency-stepped chirps," in Proceedings of the Asia-Pacific Conference on Synthetic Aperture Radar (APSAR '09), pp. 103107, Xi'an, China, October 2009.

[19] F. Parrini, F. Papi, and M. Pieraccini, "An ultra high resolution stepped frequency GPR for civil engineering applications," in Proceedings of the 8th International Workshop on Advanced Ground Penetrating Radar (IWAGPR '15), pp. 1-4, Florence, Italy, July 2015.

[20] F. Prodi and E. Tilli, "Motion compensation for a frequency stepped radar," in Proceedings of the International Waveform Diversity and Design Conference (WDD '07), pp. 255-259, IEEE, Pisa, Italy, June 2007.

[21] S. M. Wu, G. A. Ybarra, and W. E. Alexander, "Generalized optimal signal processing algorithm for frequency-stepped CW data," in Proceedings of the IEEE MTT-S International Microwave Symposium, vol. 3, pp. 1019-1022, Orlando, Fla, USA, May 1995.

[22] P. P. Patnaik, K. Arunachalam, and C. V. Krishnamurthy, "Design and validation of slot spiral antenna for stepped frequency ground penetrating radar," in Proceedings of the 17th International Radar Symposium (IRS '16), pp. 1-4, Kraków, Poland, May 2016.

[23] C.-B. Yin, X. Jia, and W.-D. Qin, "Frequency-domain extracted IFFT method for synthetic aperture radar imaging with frequency coded waveform," in Proceedings of the International Symposium on Information Science and Engineering (ISISE '08), pp. 320-325, IEEE, Shanghai, China, December 2008.

[24] D. Feng, H. Xie, D. An, and X. Huang, "A fast back-projection algorithm for bistatic forward-looking low frequency UWB SAR imaging," in Proceedings of the 16th International Radar
Symposium (IRS '15), pp. 1125-1140, Dresden, German, June 2015.

[25] L. Zhang, H.-L. Li, Z.-J. Qiao, and Z.-W. Xu, "A fast BP algorithm with wavenumber spectrum fusion for high-resolution spotlight SAR imaging," IEEE Geoscience and Remote Sensing Letters, vol. 11, no. 9, pp. 1460-1464, 2014.

[26] Y. F. Shao, R. Wang, Y. K. Deng et al., "Fast backprojection algorithm for bistatic SAR imaging," IEEE Geoscience and Remote Sensing Letters, vol. 10, no. 5, pp. 1080-1084, 2013.

[27] V. T. Vu, T. K. Sjogren, and M. I. Pettersson, "SAR imaging in ground plane using fast backprojection for mono- and bistatic cases," in Proceedings of the IEEE Radar Conference (RadarCon '12), pp. 184-189, Atlanta, Ga, USA, May 2012.

[28] L. M. H. Ulander, H. Hellsten, and G. Stenström, "Syntheticaperture radar processing using fast factorized backprojection," IEEE Transactions on Aerospace and Electronic Systems, vol. 39, no. 3, pp. 760-776, 2003.

[29] Y.-H. Li, Q. Song, P.-Y. Wang, T. Jin, and Z.-M. Zhou, "Multistage multi-resolution fast back-projection imaging algorithm," Acta Electronica Sinica, vol. 39, no. 3, pp. 518-524, 2011.

[30] H.-L. Li, L. Zhang, L. Yang, Y.-C. Li, M.-D. Xing, and Z. Bao, "A novel fast factorized back-projection algorithm for synthetic aperture radar imaging," Journal of Electronics \& Information Technology, vol. 35, no. 6, pp. 1435-1441, 2013.

[31] H.-L. Li, L. Zhang, M.-D. Xing, and Z. Bao, "Innovative strategy for stripmap SAR imaging using fast factorized backprojection," Journal of Electronics \& Information Technology, vol. 37, no. 8, pp. 1808-1813, 2015.

[32] S.-S. Zuo, Z.-M. Yang, G.-C. Sun, and M.-D. Xing, "Geometric correction based fast factorized back projection algorithm for spotlight SAR imaging," Journal of Electronics and Information Technology, vol. 37, no. 6, pp. 1389-1394, 2015.

[33] Z. Yang, Q. Dong, G. Sun, and M. Xing, "A fast implementation method for the FFBP algorithm," in Proceedings of the IEEE International Radar Conference (RadarCon '15), pp. 411-414, Arlington, Va, USA, May 2015.

[34] S. I. Kelly and M. E. Davies, "A fast decimation-in-image backprojection algorithm for SAR," in Proceedings of the IEEE Radar Conference (RadarCon '14), pp. 1046-1051, IEEE, Cincinnati, Ohio, USA, May 2014.

[35] S. Zuo, G. Sun, M. Xing, and W. Chang, "A modified fast factorized back projection algorithm for the spotlight SAR imaging," in Proceedings of the 5th IEEE Asia-Pacific Conference on Synthetic Aperture Radar (APSAR '15), pp. 756-759, Singapore, September 2015.

[36] Z. Yang, M. Xing, Y. D. Zhang, G. Sun, and Z. Bao, "Factorised polar-format back-projection algorithm," IET Radar, Sonar and Navigation, vol. 9, no. 7, pp. 875-880, 2015.

[37] D. Ran, C. B. Yin, and X. Jia, "A data block partition based fast factorized BP algorithm for bistatic airborne SAR," in Proceedings of the EUSAR 2016, pp. 363-366, Hamburg, Germany, 2016.

[38] C. Yin and D. Ran, "Converse beam cross sliding spotlight SAR imaging processing with data-blocking based fast back projection," in Proceedings of the IEEE International Geoscience and Remote Sensing Symposium (IGARSS '16), pp. 1070-1073, Beijing, China, July 2016. 


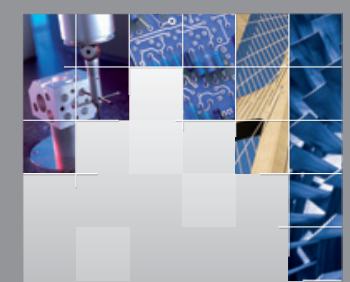

\section{Enfincering}
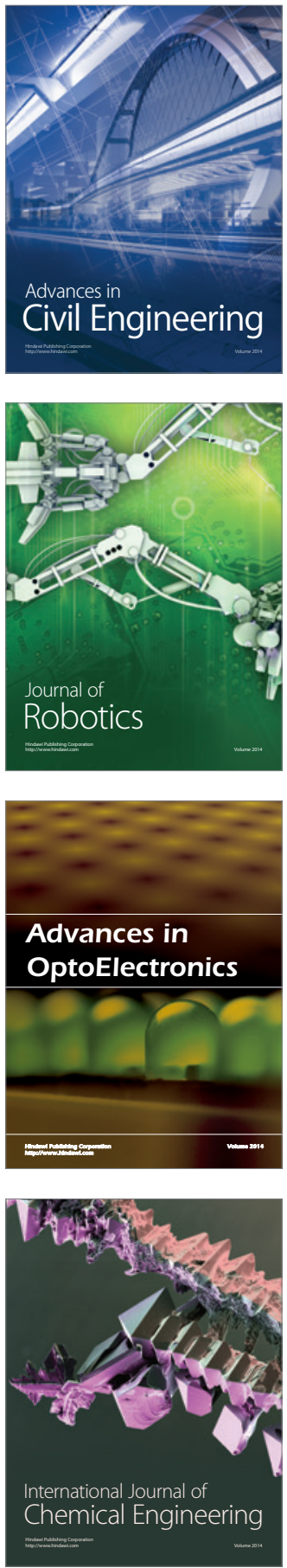

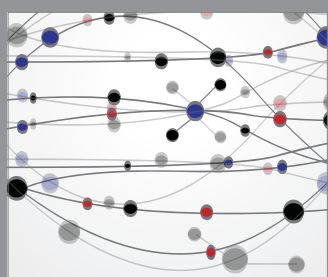

The Scientific World Journal

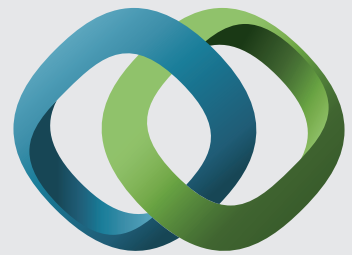

\section{Hindawi}

Submit your manuscripts at

https://www.hindawi.com
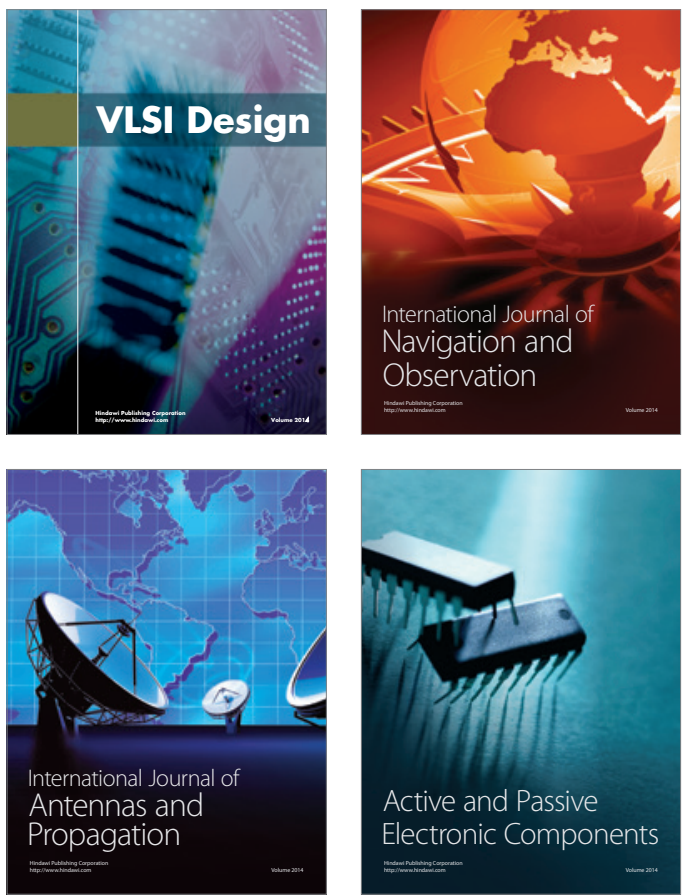
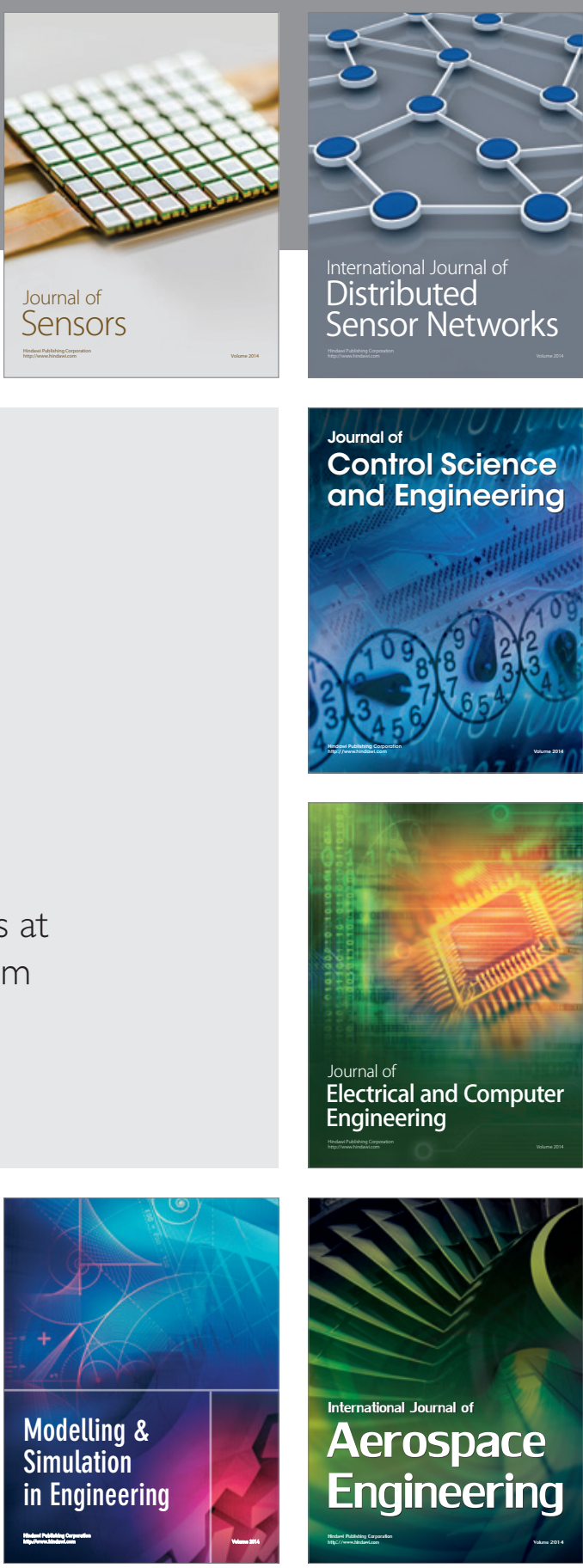

International Journal of

Distributed

Sensor Networks

$-$

Joumal of

Control Science

and Engineering
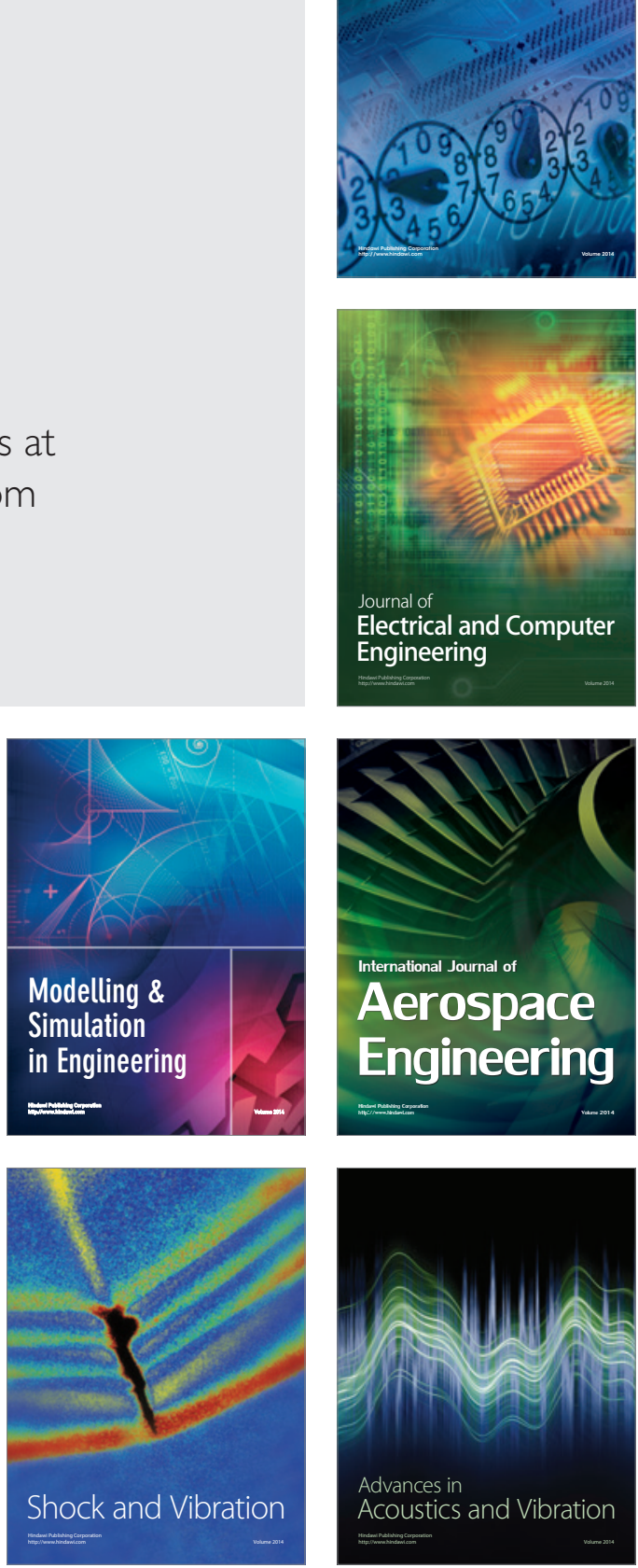